\title{
Perceptions About and Responses to Intimate Partner Violence in the Sinhalese Immigrant Community in Toronto
}

Sepali Guruge*

Daphne Cockwell School of Nursing, Ryerson University, Toronto, Ontario, Canada

*Corresponding author: Dr. Sepali Guruge, Daphne Cockwell School of Nursing, Ryerson University, Toronto, Ontario, Canada; Tel: 416-979-5000, Ext. 4964; Fax: 416-979-5332; E-mail: sguruge@ryerson.ca

Received date: March 17, 2014, Accepted date: July 16, 2014, Published date: July 23, 2014

Copyright: (C) 2014 Guruge S. This is an open-access article distributed under the terms of the Creative Commons Attribution License, which permits unrestricted use, distribution, and reproduction in any medium, provided the original author and source are credited.

\begin{abstract}
Intimate partner violence is a serious human rights issue and a critical health problem for women worldwide. Many studies have advanced our knowledge about this issue, but few have focused on immigrant communities in Canada. This paper presents the findings of a recently completed qualitative interpretive descriptive study involving seven Sinhalese women and two service providers in Toronto, Canada. An intersectionality approach was used to explore their views about intimate partner violence in the Sinhalese community. Participants acknowledged various forms of intimate partner violence within their community and identified patriarchal expectations, post-migration changes in socio-economic status, and relationship problems as contributing factors. Participants noted that most women remained with their abusive husband as a result of various micro-level (e.g., children's future), meso-level (e.g., community pressure), and macro-level factors (e.g., limitations in Canadian health, social, and settlement services). A complex interaction between gender, race, and class relations in pre- and post-migration settings appear to make women vulnerable to intimate partner violence as well as shape their responses to it. Further research is needed to develop a more comprehensive understanding of this interaction.
\end{abstract}

Keywords: Canada; Immigrant women; Intersectionality approach; Intimate partner violence (IPV); Perceptions of IPV; Qualitative methods; Responses to IPV; SINHALESE women; Sri Lanka.

\section{Introduction}

Intimate partner violence (IPV) refers to a pattern of physical, sexual, and/or emotional abuse by a current or former intimate partner or spouse in the context of coercive control [1]. While men can be victims of IPV [2], women comprise the overwhelming majority of victims of IPV across all communities, societies, and countries. IPV is linked to a range of physical and mental health problems that may persist long after the violence has ended [3], and has received increase in research attention. However, few studies have focused on how immigrant women in Canada perceive IPV, and what factors influence their responses to IPV. Perceptions of, and responses to, IPV are shaped socially, economically, culturally, and politically. Intersecting factors such as gender roles, relationship status, employment, family relations, social support, and community beliefs about IPV, as well as the availability of, and access to, appropriate care and services can influence women's vulnerability to IPV, as well as their response. Migration poses additional challenges to individuals and families, and can affect the dynamics of these intersecting factors. For example, challenges such as economic insecurity, family separation, social isolation, language barriers, discrimination, and gendered-migration policies may increase immigrant women's vulnerability to abuse. This paper discusses perceptions of and responses to IPV within the Sinhalese community in Toronto based on a recent study involving a group of Sinhalese immigrant women and service providers who spoke about the topic. This is the first study to focus on IPV in this community in Canada.

\section{Literature Review}

At a global level, IPV occurs in epidemic proportions despite increases in clinical, research, and policy changes to address this issue. The recent World Health Organization (WHO) study reported that the rates of physical and/or sexual violence among ever-partnered women $(\mathrm{N}=24,000)$ varied widely $(15-71 \%)$ across 10 countries, as did women's responses to IPV [4]. Canada's well-known Violence Against Women Survey (1993), which included 12,300 women who were randomly interviewed, revealed that $10 \%$ of Canadian women (18 years or older) had experienced violence in the 12 months preceding the survey, and that $51 \%$ had experienced physical or sexual assault since the age of 16 [5]. Data from the 1999 General Social Survey (GSS) [6], which included 20,000 participants of which 14,269 were women (aged 15 or older) from 10 provinces, indicated that approximately $8 \%$ of Canadian women in married or common-law relationships experienced abuse from their partner on at least one occasion during the 5-year period prior to the survey, and that $37 \%$ of the women who had ever been married or in a common-law relationship had experienced at least one incidence of IPV.

Very little information is available about IPV prevalence in immigrant households in Canada. Cohen and Ansara [7] analyzed 1999 GSS data and found that IPV rates (for both severe and nonsevere forms of violence) tended to be lower among newcomer women (less than 10 years in Canada) than among long-term immigrants or Canadian-born women. Using the same dataset, Ahmad et al. [8] found that new immigrant women reported significantly more emotional and financial abuse than Canadian-born women did $(14.7 \%$ and $9.3 \%$, respectively), but found no statistically significant differences in rates of physical abuse. Based on 2006 GSS data, Sinha [9] reported that $4.9 \%$ of Canadian immigrant women self-reported being a victim of IPV in the previous five years. However, these rates 
should be interpreted with caution as the GSS had several limitations: (a) it was a telephone survey, so it excluded those without phones; (b) it excluded those who did not speak English or French; (c) it measured only violence that had occurred during the last 12 months and during the 5-year period prior to the survey; (d) it did not measure certain forms of violence such as sexual abuse; and (e) it may be subjected to reporting bias, as some women might have been hesitant to respond (or respond accurately) to this kind of sensitive topic in a telephone survey.

Several Canadian studies have focused on the topic of IPV in the post-migration context $[8,10-13]$. Based on a meta-synthesis of Canadian health sciences studies on the topic, Guruge et al. [14] found commonalities among the contributing factors of IPV across nine immigrant communities: loss of, or limited, social networks and support; changes in gender and sexual relationships; and, changes in socioeconomic status. These changes tended to negatively affect the marital relationship, often leading to separation, divorce, family conflicts, and IPV. The findings of these studies also highlight the importance of understanding perceptions about IPV, the determinants of IPV, and what influences women's responses to IPV in various communities. For example, Guruge and Humphreys [15] reported many systemic barriers to help-seeking among Tamil immigrant women in Canada. Clarifying these issues will be vital to the development and implementation of appropriate practice and policy changes to promote healthy marital relationships to prevent IPV, and help women deal with IPV in the post-migration context in Canada.

Studies on immigrant women in other countries such as the US [16-18], Norway [19], and Hong Kong [20] report some commonalities and differences between immigrant and nonimmigrant women's approaches and responses to IPV and its consequences. For example, Amanor-Boadu et al.'s [17] study about non-immigrant and immigrant women, including South Asian women in New York, demonstrated that immigrant women face more barriers to leaving their abusive partners than their non-immigrant counterparts. For example, even after leaving their partners, immigrant women were less likely to be able to go somewhere that their partners could not find them. Immigrant women participants were also more likely to report feeling socially isolated, controlled by their partners, and afraid of their partners than non-immigrant research participants. Wong et al. [20] reported that Chinese women who immigrated to Hong Kong and experienced IPV there were more depressed and perceived less social support than Hong Kong-born women who had experienced IPV. They also reported that while depression as a health consequence of IPV is not specific to immigrant women, it lasts longer in immigrant women. Other factors specific to the experience of post-migration context included language barriers, lack of knowledge about the local community services [21], and not having usually safe places (i.e., parents or relatives' home) to escape from abuse [22].

While commonalities exist across groups of women for not seeking help if they experience IPV, there are also factors related to specific socio-cultural groups. For example, Lee et al. [23] reported that unfavourable attitudes among older Korean immigrant women in the US towards reporting IPV and their help-seeking were similar to their counterparts in Korea; they explained that this is related to the vital role of cultural and social factors such as within-group loyalty, "saving face," and keeping family matters inside the family, rather than an immigration effect on women's responses to IPV. Studying IPV among Spanish-speaking immigrants in the US, Beauchamp and colleagues [24] also concluded that sharing a common language, heritage, and culture can lead to sharing attitudes and beliefs about IPV. Cultural attributes that shape attitudes toward family were perceived as both risk and protective factors among Hispanic immigrants in the US [25]. In a study about South Asian women who had experienced IPV in the US, Kallivayalil [26] reported that both migration and culture can have a deep impact on the personal experience of suffering. Horn [22] study with participants in a refugee camp in Kenya reported that the community's attitude toward IPV influenced whether a woman would report IPV, and whether she would be blamed or her whole family stigmatised if she reported. In Shoultz et al.'s [16] study with Filipino immigrants in Hawaii, not reporting IPV was related not only to a fear of bringing shame to the family but also to avoid damaging their community's reputation within the larger society.

\section{Community of Focus}

This paper focuses on Sinhalese women in Toronto, Canada, where Sri Lankans form a considerable portion of the immigrant population. Over the last three decades Sri Lanka has undergone a sizeable exodus, largely due to the 25 year civil war that began in 1983 and subsequent political and social changes. In 2010, 1,847,500 $(9.1 \%$ of the population) emigrated from the country, and Canada was one of the top destination countries [27]. The Sri Lankan community in the Greater Toronto Area consists primarily of two distinct ethnic groups: Tamils (approximately 300,000 individuals) and Sinhalese (approximately 50,000 individuals). Several studies have focused on IPV among Tamil immigrants from Sri Lanka [12,28-30] however, no studies to date have focused on IPV in the Sinhalese community in Canada. Focusing on the Sinhalese community is important given the social, cultural, religious, and other differences (such as primary reason for migration) between the two communities.

\section{Purpose}

The purpose of this study was to explore the perceptions of, and responses to, IPV in the Sinhalese community. The overall research question was: What are women's perceptions of, and responses to, IPV in the Sinhalese community of Toronto?

\section{Theoretical Framework}

The study was guided by an intersectionality perspective [31,32], which helps clarify the complexity surrounding multiple axes of social identity (such as gender, race, class, ethnicity, and culture) and how these intersect in distinct ways to influence the health and wellbeing of individuals and/or groups [33]. By exploring multiple and multi-level factors and their interactions, this perspective can help identify broader issues such as racism, sexism, and classism as well as various institutional and structural elements that maintain inequalities between various groups of women and men [33]. This kind of approach to research is especially important in the area of IPV in the post-migration context, because it can help reveal the diverse forms of oppression experienced by women and clarify how gender relations intersect with race, ethnic, class, and sexual relations in the postmigration context to create a disproportionately higher risk of IPV, or more difficulty dealing with IPV, among some individuals and groups. 


\section{Methods}

A qualitative interpretive descriptive design [34] was used in this study. This approach helps advance knowledge through a process of exploration grounded in the experience of individuals; it also complements the intersectionality approach that guided this study and focuses on diversity within and between groups, shared experiences within and across groups, and the contextual factors beyond individual actions/actors that create diverse forms of oppression.

Following ethical approval from Ryerson University, participants were recruited using a snowball sampling strategy. Inclusion criteria were: Sinhalese women immigrants, 18 years or older, living in the Greater Toronto Area, and willing to talk about their own and/or others' experience of IPV and/or about what is going on in the community regarding IPV. Interviews were held at a time and location that was convenient for each participant and in the participant's preferred language (most chose Sinhalese). Participants were informed (via a written consent form and verbally) of their right to decline participation, to refuse to answer any question, and to terminate the interview at any time. Interview questions focused on issues related to gender, race, and class relations, family conflict, and IPV in the community in the post-migration context. Adjustments to questions were made as the interviews progressed, based on completed interviews. Each participant was interviewed only once, and each interview lasted 1-2 hours. With consent, the interviews were audiorecorded. An honorarium ( $\$ 30$ ) was given to each participant to defray the costs of childcare, transportation, and time.

Interviews that were conducted in Sinhalese were translated into English and transcribed by a research assistant, and randomly checked for accuracy by the author. Using a sentence-by-sentence process, each interview was then coded and sorted/organized using NVivo, and significant codes were grouped as subcategories. The subcategories already developed were compared with the new codes to determine commonalities and variations, and to develop categories [35].

Trustworthiness of the study [36] was ensured through several strategies: interviewer triangulation (interviews were conducted by two interviewers); interviews conducted in two languages (to capture opinions of both English- and non-English-speaking participants); member-checking (with each participant during each interview, and others during subsequent interviews); and careful documentation (an audit trail) of the procedures, rationale for, and congruency in the development of codes, subcategories, and categories, and the conclusions that were drawn.

\section{Results}

It was difficult to recruit women who had experienced IPV and were willing to speak about their own experience of IPV. Therefore, the final sample included seven Sinhalese women living in the Greater Toronto Area (who knew other Sinhalese women who had experienced IPV) and two service providers who had worked with abused Sinhalese women), who were willing to speak about what is going on in this community. Thus, the participants took on a key informant role in this study.

The Sinhalese women (noted as 'SW' below) participants were diverse in terms of age (19-51 years), education (Grade 10-Bachelor's degree), marital status (single, married, and widowed), length of residency in Canada (three months-16 years), and employment in Sri Lanka (two students; all others were in paid employment) and in
Canada (two were in college/university, two in paid employment, and three unemployed). The two service providers (noted as 'SP') were female and also from Sri Lanka (one Sinhalese and one Tamil); they were in their mid-40s and mid-50s, were both married with children, and had both been working in the field of settlement in Canada for more than 10 years. One of the service providers was interviewed at the start of the study to help obtain an initial impression of the topic, and the second was interviewed at the end of the study to help fill in any gaps in the emerging picture.

The next sections present four main areas of findings: perceptions of IPV, determinants of IPV, responses to IPV, and determinants of women's responses to IPV.

\section{Perceptions of IPV}

Based on their knowledge of what was going on in the Sinhalese community in the Greater Toronto Area, participants referred to various behaviours and actions by husbands that constituted forms of IPV: physical, verbal, emotional, sexual, and financial abuse, and other forms of threats and coercive control. The following quotes illustrate some of these forms of IPV:

They have been going through physical abuse... really bad physical abuse. (SP \# 1)

He puts her down a lot after coming to Canada. He uses words that are really demeaning to women and sometimes he uses words such as bitch. (SW \# 1)

A lot of the times the women have to be employed [in Canada]. If she gets delayed at work by an hour the husband questions her on it. She is petrified to come home. (SW \# 6)

After coming to this country, men get exposed to so many new things and new ideas, and with the freedom available here, they start to experiment and expect too many sexual stuff from the women. This leads to sexual abuse. (SP \# 2)

There was a time he didn't give her any money for no reason. (SW \# 4)

The husband used to threaten her a lot if she found out facts about something and had a different point of view. (SW \# 7)

Just like that he asks her to shut up ... he would say, 'I am going to smash your head' (SP \# 1)

There is this guy I know he doesn't allow his wife to use the Internet. She really wants the Internet because she gets news about home from the Internet. (SW \# 5)

Of all the forms of IPV listed by participants, emotional abuse was noted as the most common form; this included threats of physical violence, and constant belittling and demeaning of women. Participants also indicated that men used verbal threats, restrictions of movement, and constant checking up on women, as well as limiting their contact with the outside world; all of these are features of an extreme level of control exerted by abusive husbands [37].

\section{Determinants of IPV}

According to the participants, IPV generally began after couples immigrated to Canada. A number of interrelated determinants of IPV emerged from the interviews: husband's patriarchal expectations of his 
wife, post-migration changes in socio-economic status, and relationship problems such as extramarital affairs.

Husbands' expectations of wives had been shaped by gendered norms (seen in almost all societies around the world), which promote (to varying degrees) women's subordinate status relative to men. Participants said that ideas about a 'good obedient wife' and the 'male head of the household' were generally ingrained into women and men from childhood, and that these norms were perpetuated within this community in Canada by both men and the women:

Here [in Canada], both partners come home [from work] at the same time and they are both tired but the wife's duties aren't finished, and she has to clean and do everything at home. The men are used to the traditional ways and are not willing to compromise. For example, the men expect to have hot, hot rice and they are unwilling to eat leftovers. In addition, she has to take care of the children, their school needs, and other such things. (SW \# 7)

Sinhalese family setup is such that the man is seen as the head of the family ... the patriarch! A lot of men try to assert their power on the relationship and they feel that the wife has to listen and accept everything he says. (SW \# 3)

Although these patriarchal beliefs and expectations appear to be changing among Sinhalese couples in Sri Lanka as women gain more access to employment and freedom to travel within and outside the country [38], the participants felt that most men in their community continued to maintain an unequal distribution of power at home. When men's expectations regarding traditional gender roles and responsibilities were not fulfilled or caused conflict, women were at increased risk of abuse by their husbands.

In all cases of IPV described by Sinhalese women and service providers, the husbands had experienced negative changes in socioeconomic status since coming to Canada, often due to un/underemployment, lack of recognition of credentials, and deskilling. These changes often led to a loss of social status at home and in the community creating frustration, depression, anxiety, anger, and aggression among the men, which was perceived by the participants as leading to family conflict and IPV. The following excerpts reveal some of these ideas:

They were facing all sorts of hardships as a result of this financial situation. They were living in a really bad basement here. In Sri Lanka they had a huge house with seven rooms and three baths, several vehicles, and drivers and servants. They did not want to go back because of children's future. But he was getting more and more frustrated with the situation and taking it out on his wife. (SP \# 2)

If she sees someone else having something she wants it, even if he says that it is not a good time to buy things for whatever reason. So, he is struggling to maintain a lifestyle but this gets out of hand and leads to abuse when he cannot maintain a certain lifestyle. (SW \# 6)

The patriarchal ideology that governs most marriages worldwide dictates certain gender relations, expectations, and role fulfilment for husbands as well. As is the case in many communities, Sinhalese husbands are expected to be the main breadwinner of the family, and their self-worth may be affected by their ability to secure employment and financially support the family. This can be problematic in the post-migration context, due to the frequently discriminatory and racist employment sector in Canada [39] where most immigrant men and women are underemployed and underpaid [40].
Participants identified relationship problems such as jealousy, suspicion, and extramarital affairs as determinants of IPV in their community. Patriarchal expectations often involve different standards for men and women, and women may be punished more severely for committing the same act [12]. In many South Asian communities, including the Sinhalese community, substance abuse, sexual indiscretion, and other risk-taking behaviors tend to be more acceptable for men [41]. In contrast, Sinhalese women are taught that virginity and virtue are essential characteristics, and society condemns women who have intimate relationships prior to marriage or outside the marriage [41]. This double standard continues in the postmigration context, affecting women disproportionally and placing them at risk for IPV. The following excerpts capture the general perception among the participants:

I think they [men and women] find here a freedom that they did not have in Sri Lanka, and they take advantage of that and go out of control. Men having extramarital relationships and divorcing the wife ... sometimes both partners have had affairs, but the woman gets abused when she is suspected of having an extramarital relationship. (SW \# 5)

So there are extramarital relationships here [in Canada]. I think that as humans it is very natural for her to have such an affair when she was all alone here but in our community it is difficult to move past such a thing. (SW \# 4)

Participants said that affairs often began when husbands and wives were separated due to sponsorship delays or the husband finding employment elsewhere because of challenges in securing jobs in Canada at the level of their education, training, and skills level. Some participants also commented that Canada's social and cultural context did not involve the societal monitoring and control found in Sri Lanka, thereby providing more freedom for extramarital relationships. Regardless of who had the affair, in situations of extramarital relationships women were perceived as being at greater risk for IPV.

\section{Women's Responses to IPV}

In most contexts, disclosure and recourse are often not considered by abused women [42-45]. Participants in this study also noted that abused women tended to suffer in silence and find ways to live with the abuse:

They are just keeping quiet and suffering. They are not ready to come out of the family system. For example, she says she can't leave because she loves him a lot. (SP \# 1)

There is a notion that you cannot live without a husband and you feel the need for a sense of security provided by a husband. (SW \# 1)

She believes it is a good thing to be patient and figure out ways to remain in the marriage because if you hold on through a hard period, chances are that things will get better. It is best to keep a marriage together for the sake of the children. (SW \# 4)

Both groups of participants noted that women often struggled with the decision to call the authorities or leave an abusive husband. Separation and/or divorce were considered only by a minority of abused women:

The wife called 911 and the husband was put in jail and he could not go home. She said that it was better for her child that they were living separately than having to see her parents fight all the time. (SW \# 3) 
Participants identified several determinants of women's responses to IPV, which can generally be located at three levels of society: micro-, meso-, and macro-levels.

Micro-level (individual and family level) factors included children's wellbeing, women's financial dependency on the husband, family welfare, and friends' responses.

According to participants, one key determining factor in a woman's decision-making process is her children's safety, comfort, and future. As in many communities and societies, Sinhalese women believe that it is important for children to have a strong paternal presence and influence in their everyday lives:

They cannot think about their own wellbeing and walk away from the husband and jeopardize their children's future. (SP \# 2)

This belief is further reinforced by family and friends:

If she has very young children I will recommend that they go see a counsellor. I suppose she can get a separation if her life is threatened. (SW \# 4)

We told them that every family has problems and if you harp on those problems it is the children who are going to suffer. A child needs a mother and a father. (SW \# 3 )

Only a few participants referred to how a father's violence toward their mother might affect the children and their overall wellbeing.

Another key determinant of women's responses as perceived by the participants was financial dependency on the husband. Many Sinhalese women in Canada are engaged in paid labour, but they are often under-employed and under-paid, as are many women in other immigrant communities [40], and their pay is insufficient to start a separate life for themselves and their children. Women's financial status is also dependent on their migration trajectory:

Especially when the husband is the first to come to Canada, the wife and the children are dependent on him. She cannot financially separate from him. (SW \# 1)

Participants said that most Sinhalese women tend to be more financially dependent on their husbands in the post-migration context - preventing them from taking advantage of the programs and services (such as shelters) available in Canada to leave an abusive husband. Thus, both patriarchy and racism intersect in Canada to make Sinhalese immigrant women (as are immigrant women from other racialized immigrant communities $[11,12]$ even more vulnerable when facing IPV.

According to our participants, women's responses to IPV were also heavily influenced by their extended families' welfare:

When women get abused they don't even tell their parents about it because they do not want them to worry. (SW \# 1)

Her mother-in-law tells her to take care of her son, and she says that this is his nature and that she should try to hold on to him. There is a lot of pressure for her to remain in the marriage, and she keeps thinking that her parents are going to feel bad, and the mother-in-law is going to suffer. She is not thinking about her and her children. (SP \# 1)

In collectivist societies, individuals (especially women) tend to focus on the welfare of the family when considering marriage, separation, and divorce; women are often expected to sacrifice their own welfare for that of their families [41]. This is especially true when it comes to the woman's own family (i.e., parents, siblings etc.), but participants highlighted cases (as is the case in the above excerpt) where the abused women also considered the wellbeing of their parents-in-law when making decisions.

Friends' responses also can have a considerable influence on a woman's decision-making process, as shown by the excerpts below:

I would tell her to correct her mistake because that is what leads to the abuse. I will tell him to think of his children and find an alternative to soothe himself if he is tired. (SW\# 5)

I told her: You have to help him find other ways to get over his anger because it is the anger that leads to abuse...no matter what comes your way you have to forget and move on (SW \# 6)

These excerpts reveal that friends' responses tend to support traditional gender roles and responsibilities for women, apply normative standards to the abuse, and blame the women for the abuse, all of which are shaped by traditional gender, family, and marital relations grounded in patriarchal ideology.

Participants identified several meso-level (community level) factors, including: social apathy regarding violence, which reinforces the belief that IPV is a private matter; community pressure for women to remain in their marriage; and/or community support (or lack thereof) for establishing a new life in the post-migration context. A key meso-level factor, community pressure to remain in the abusive relationship, was related to pressure to avoid social stigma and maintain social status within the community:

Women keep the abuse to themselves. We are a community that tries to hide our problems. We don't discuss personal matters even with our friends because we feel that we will lose our status in the community, so, we bottle it all inside. (SW \# 3)

Women try to hold on to their marriage and suffer through many things because of our culture. It is ingrained in us these things should be kept in the family. (SW \# 4)

Participants also noted that the belief that IPV is a private matter and should be hidden from the community tends to be reinforced by perceived competition and jealousy in the community:

Most of the time they [community members] are jealous of each other. They might talk to us nicely but they don't tell us where to get help or access to jobs. A Sinhalese person would not help another Sinhalese person. There is competition between families. (SW \# 2)

The pressure to hide problems in the post-migration context seems to be linked with saving face and/or maintaining social status. There appear to be two seemingly contradictory forces at play - while some participants acknowledged the many supports they received from other Sinhalese immigrants, this support seems to be limited to certain domains of their life in Canada. Many had lost much of their premigration economic status, and felt pressured to hide the problems from each other to rebuild and/or maintain their social status within the community. This was perceived as shaping the kind of support offered to others. In addition, participants perceived that maintaining community status (as a model community) within the larger society is considerably important to this (racialized) community.

The macro-level factors identified by participants were generally associated with limitations in health, social, and settlement services. Similar to previous findings about Sri Lankan Tamil immigrant women $[15,46]$, Sinhalese immigrant women participants reported 
that access to services for IPV was dependent on the acceptability of the services offered, language barriers, and lack of/limited access to information about available services. Some of these ideas are captured in the next few excerpts:

There aren't any support services targeted to help Sinhalese people. So, when they have these problems, they keep it to themselves. I don't think that we have a place to send the abused women. There should be a place where they can go and share problems, find a place to live, get access to health services and schooling for children. We need people who speak our language to direct us to support services. (SW \# 3)

There might be services but if women do not know about them, they are not useful. For example, I know I can call 911 but I don't know exactly for what emergencies. (SW \# 2)

When I asked her why she didn't call me before to talk about the abuse, she told me that it is because of the cost of the phone calls. I said there is no cost for local phone calls. (SW \# 5)

Participants also referred to other limitations to services. For example, the lack of formal support addressing determinants of IPV (e.g., women's financial dependency on the husband, friends' responses, and community pressure) was seen as an important gap by participants; they believed that having assistance to overcome these difficulties would help prevent IPV and/or enable women to leave their abusive husbands:

Most of these problems [abuse] begin because of the stresses we go through. What we need is help at the beginning to find jobs, rentreduced places to stay, low-cost daycare, and crash courses to get Canadian knowledge and job placement. (SW \# 1)

Interestingly, most participants' recommendations to prevent IPV and/ or to help women deal with IPV involved addressing various macrolevel factors such as access to employment and fair wages, rent-reduced housing, low cost day care, and social support, all of which are currently lacking or limited due to intersecting gender, race, ethnic and class relations in Canadian society.

\section{Discussion}

The findings presented in this paper revealed the complex, multilevel factors that influence how Sinhalese immigrant women perceive and respond to IPV. In many ways the results are similar to the experiences of other immigrant and non-immigrant women in Canada, but the findings also revealed unique social, economic, cultural, political, and racial elements that intersect with individual, family, community, and societal relations in the pre- and postmigration contexts for the Sinhalese immigrant community in the Greater Toronto Area. These findings are discussed next.

Participants in this study described various forms of IPV experienced by Sinhalese women in the Greater Toronto Area, including physical, verbal, sexual, and financial abuse. They also described emotional abuse and coercive control, both characteristic of violence in intimate relationships. Similar findings were reported by Guruge [47] and Hyman et al. [48] within the Sri Lankan Tamil community in Toronto as well as in other immigrant $[8,10,13]$ and non-immigrant communities in Canada. The participants in the current study perceived that IPV is a private and shameful topic and women in their community tended to hide their experience of it. This may have contributed to the challenges we faced in recruiting participants to talk about their own experiences of IPV (especially given that most of the interviews were conducted by the author and an RA who are Sinhalese). However, all participants knew of other Sinhalese women who have experienced, or were living with, IPV at time of the interviews and were able to speak in detail about these women's experiences of IPV.

Participants tended to believe that IPV began only after couples immigrated to Canada, and identified a number of interrelated determinants of IPV: husband's patriarchal expectations of the wife, post-migration changes in socio-economic status, and suspicion of or actual extramarital affairs. However, some of these factors, especially suspicions of infidelity [42,49], sexual jealousy, and resultant marital dysfunction have been identified as factors contributing to IPV in the Sri Lankan context as well [42,49,50-52]. Additional risk factors noted in these studies included: financial problems, alcohol use/abuse, women's (lower) level of education, social class, and "anxious or impulsive personality." In addition, previous research about Sinhalese couples in Sri Lanka has reported that IPV tended to occur as a result of the husband's desire to pre-empt any challenge or threats to the power structure within the family due to changes in gender relations [53], particularly related to women's improved access to education and employment, and travel away from the home.

However, these challenges are likely even more significant in the post-migration context, where men's expectations are continually challenged in a different social, economic, and cultural environment, and where women have and/or are perceived to have more freedom. A meta-synthesis of IPV research involving immigrants living in Canada [14] revealed that the downgrading of immigrants' skills, unemployment and underemployment, and the resulting changes in socioeconomic status is a common social inequity in Canada that contributed to IPV across nine immigrant communities. Within the context of the resultant poverty and other intersecting post-migration factors such as minority status, racialized status, lack of formal and informal support, and role reversals, some men may turn to self- and family destructive behaviours such as gambling, alcohol use/abuse, extra-marital relationships, and IPV [54-58]. This highlights how the patriarchal ideology that dictates certain gender, class, and sexual relations at various levels of society, along with class relations, intersect with race relations in the post-migration context in Canada to create an even greater vulnerability among racialized immigrant women to IPV.

One common finding across settings is women's reluctance to seek recourse. Literature in Sri Lanka about IPV (see for e.g., [43]) demonstrates that women's responses to IPV vary widely and are affected by their socioeconomic, geographical, cultural, and political situation. However, women most often tolerated the abuse regardless of their cultural and religious backgrounds. When they did seek help, the support primarily came from their mothers. Other family members (both immediate and extended) helped by giving advice, offering shelter, giving food, and also assaulting the abuser. However, such support is rare in the post-migration context. Sinhalese women living in the Greater Toronto Area were thought to suffer in silence and to find personal ways of coping with abuse, with most opting to remain in abusive relationships. Similar responses have been observed among the Tamil community in Toronto [47].

Previous research about IPV in Sri Lanka and in other countries has also discussed the importance of factors such as the nature and severity of abuse, education level, informal social networks and support, and accessibility and cultural acceptability of formal support agencies, in determining women's responses to IPV $[44,58,42,46]$. Specifically, 
women's responses to IPV are determined by intersecting factors at the micro-, meso- and macro-levels. Micro-level determinants, such as children's wellbeing, a woman's financial dependency on the husband, and expectations and responses reinforced by family and friends (primarily through socially-sanctioned gender inequities, male domination, primacy of heterosexual relationships, and the importance of paternal influence of children), were similar among immigrant Tamil women in Toronto [46], non-immigrant Sinhalese women in Sri Lanka [43], and Sinhalese women in the Greater Toronto Area (as is the case in most other communities and societies in the world). Within communities where patriarchal norms are more pervasive across all levels of society, women are expected to be subservient and tolerant, and sacrifice their wellbeing for the benefit of their husband and children, which limits their financial independence and reduces options for separation, divorce, and remarriage. While some of these expectations change following migration, in some cases, the expectations are even more reinforced to ensure women's subordination. Gendered and racist immigration policies further enforce these expectations.

Meso-level determinants such as community-enforced sanctions on infidelity and double-standards for women and men, belief that IPV is a private matter, social stigma associated with divorce, and the selective support from the community are the result of intersecting gender and class relations. According to women interviewed in this study, these determinants are reinforced by perceived competition and jealously in the community and the need and pressure to rebuild and/or maintain status within the community, both of which are related to diminished socioeconomic status following migration to Canada, which, in turn, is a direct result of the racist and discriminatory practices in the immigration system and employment sector, and racism in broader Canadian society.

Macro-level determinants identified by the participants were related to limitations in the availability and accessibility of services. Given the micro- and meso-level factors noted above, women seeking to escape abusive relationships rely on health, social, and settlement services for their informational, financial, legal, health, and settlement needs. However, our participants indicated that women are often prevented from accessing these services effectively due to various systemic and structural barriers. The inadequate responses of health, social, settlement, and legal institutions to IPV among immigrant women in Canada have been documented. For example, based on research about IPV in the Sri Lankan Tamil community in Canada, Guruge and Humphreys [15] identified various limitations in the services including the following: lack of information about available services, lack of culturally-safe and linguistically-appropriate services, lack of portability and seamlessness of services, confidentiality issues, and various discriminatory and racist practices embedded in services and service delivery. When these limitations result in women remaining (or remaining longer) in abusive relationships, society at large is, in effect, making immigrant women even more vulnerable [54].

\section{Limitations}

The study involved a small convenience sample of women and service providers. Additionally, while the participants were knowledgeable about what is happening in the Sinhalese community in the Greater Toronto Area, to our knowledge, they had not experienced IPV personally. Despite these limitations, our findings provide some initial insights into IPV within this immigrant community in Canada, which has not yet received health research attention.

\section{Implications}

This study has a number of implications for research. Further research needs to be conducted with a larger sample in particular with Sinhalese women who have experienced, or are experiencing, IPV in Canada to clarify the extent to which the findings presented here are relevant across the community. Further research is also needed to clarify the nuances of why IPV seems to begin following migration to a country such as Canada. Additionally, the findings suggest that conflict (e.g., community pressure, competition, and jealousy) in women's immediate social networks and the broader community can have a significant negative influence on women's responses to IPV. This requires further exploration especially since previous research about IPV has primarily focused on the positive dimensions of ethnocultural networks. Finally, research involving other immigrant communities with a range of settlement experiences (e.g., immigrant versus refugee, newcomer versus established, larger versus smaller community, and urban versus rural settlement) will help identify unique and shared experiences within and across groups, as well as relevant contextual factors, which will ultimately help design more effective and comprehensive services and programs for immigrant women dealing with IPV in the post-migration context.

\section{Acknowledgements}

The author acknowledges financial support for the study (2007 2009) from a SSHRC Institutional Grant from Ryerson University. The author also acknowledges financial support for her work from the Institute of Gender and Health of Canadian Institutes of Health Research (CIHR) in the form of a New Investigator Award. The author is grateful to the study participants. She also wishes to thank Dr. Margaret Malone (co-investigator) and the research assistant who helped with the data collection as well as Dr. Vathsala JayasuriyaIllesinghe (who critically reviewed a previous version of the manuscript).

\section{References}

1. Tjaden P, Thoennes N (2000) Extent, Nature and Consequences of Intimate Partner Violence: Findings from the National Violence Against Women Survey. National Institute of Justice and the Center for Disease Control and Prevention.

2. Stewart DE, MacMillan H, Wathen N (2013) Intimate partner violence. Can J Psychiatry 58: Insert 1 - 15, Encart 1-17.

3. Campbell J, Jones AS, Dienemann J, Kub J, Schollenberger J, et al. (2002) Intimate partner violence and physical health consequences. Arch Intern Med 162: 1157-1163.

4. World Health Organization (2006) Multi-country study on Women's Health and Domestic Violence against Women. Summary Report.

5. Statistics Canada (1993) Violence against Women Survey (Report No. 11-001E). Government of Canada, Ottawa.

6. General Social Survey on spousal violence: A fact sheet (2000).

7. Cohen M, Ansara D (2002) Violence against women. In DE Stewart, A Cheung, LE Ferris, I Hyman, M Cohen \& IJ Williams (Editors), Ontario Women's Health Status Report (Prepared for the Ontario Women's Health Council)

8. Ahmad F, Ali M, Stewart DE (2005) Spousal-abuse among Canadian immigrant women. J Immigr Health 7: 239-246.

9. Sinha M (2013) Measuring violence against women: Statistical trends. Canadian centre for Justice. 
10. Barata PC, McNally MJ, Sales IM, Stewart DE (2005) Portuguese immigrant women's perspectives on wife abuse: a cross-generational comparison. J Interpers Violence 20: 1132-1150.

11. Fong J (2000) Silent no more: How women experienced wife abuse in the local Chinese community. Unpublished doctoral dissertation, York University, Toronto, Canada.

12. Guruge S, Khanlou N, Gastaldo D (2010) Intimate male partner violence in the migration process: intersections of gender, race and class. J Adv Nurs 66: 103-113.

13. Hyman I, Guruge S, Mason R (2008) The impact of post-migration changes on marital relationships: A study of Ethiopian couples. Journal of Comp. Family Studies 39: 149-164.

14. Guruge S, Shirpak R, Zanchetta M, Gastaldo D, Hyman I, et al. (2010) A meta synthesis of post-migration changes \& their effects on marital relationships in Canada. Canadian Journal of Public Health 101: 327-331.

15. Guruge S, Humphreys J (2009) Barriers affecting access to and use of formal social supports among abused immigrant women. Can J Nurs Res 41: 64-84.

16. Shoultz J, Magnussen L, Manzano H, Arias C, Spencer C (2010) Listening to Filipina women: perceptions, responses and needs regarding intimate partner violence. Issues Ment Health Nurs 31: 54-61.

17. Amanor-Boadu Y, Messing JT, Stith SM, Anderson JR, O'Sullivan CS, et al. (2012) Immigrant and nonimmigrant women: factors that predict leaving an abusive relationship. Violence Against Women 18: 611-633.

18. Montalvo-Liendo N, Koci A, McFarlane J, Gilroy H, Maddoux J (2013) U.S.-born compared to non-U.S.-born abused women: analysis of baseline data. Hisp Health Care Int 11: 111-118.

19. Bø Vatnar SK, Bjørkly S (2010) An interactional perspective on the relationship of immigration to intimate partner violence in a representative sample of help-seeking women. J Interpers Violence 25 1815-1835.

20. Wong JY, Tiwari A, Fong DY, Humphreys J, Bullock L (2011) Depression among women experiencing intimate partner violence in a Chinese community. Nurs Res 60: 58-65.

21. Kyriakakis S, Dawson BA, Edmond T (2012) Mexican immigrant survivors of intimate partner violence: conceptualization and descriptions of abuse. Violence Vict 27: 548-562.

22. Horn R (2010) Responses to intimate partner violence in Kakuma refugee camp: refugee interactions with agency systems. Soc Sci Med 70: 160-168.

23. Lee HY, Yoon HS, Yoon JY, Kwon JH, Park ES, et al. (2014) Perception and help-seeking intention of intimate partner violence in later life: An international perspective. Journal of Aggression, Maltreatment \& Trauma 23: 45-66.

24. Beachchamp R, Lindsay SP, Hunter LP, Talavera G (2012) Patriarchal beliefs and attitudes toward intimate partner violence among Spanishspeaking adults. Hispanic Health Care International 10: 137-145.

25. Gonzalez-Guarda RM, Cummings AM, Becerra M, Fernandez MC, Mesa I (2013) Needs and preferences for the prevention of intimate partner violence among Hispanics: a community's perspective. J Prim Prev 34: 221-235.

26. Kallivayalil D (2010) Narratives of suffering of South Asian immigrant survivors of domestic violence. Violence Against Women 16: 789-811.

27. The World Bank (2011). Migration and remittences factbook 2011. World Bank, Washington: DC.

28. Hyman I, Mason R, Guruge S, Berman H, Kanagaratnam P, et al. (2011) Perceptions of factors contributing to intimate partner violence among Sri Lankan Tamil immigrant women in Canada. Health Care Women Int 32: 779-794.

29. Morrison L, Guruge S, Snarr KA (1999) Sri Lankan Tamil immigrants in Toronto: Gender, marriage patterns, and sexuality. In G. A. Kelson \& D. L. DeLaet (Editors), Gender and immigration. New York University Press., New York.

30. Tyyskä V, Dinshaw F (2009) Families and violence in Punjabi and Tamil communities in Toronto. CERIS Policy Matters No. 39.
31. Crenshaw KW (2005) Mapping the margins: Intersectionality, identity politics, and violence against women of color. In Bergen, R.K., Edleson, J.L., Renzetti, C.M. (Editors) Violence Against Women. Classic Papers. Boston, MA: Pearson Education.

32. Hankivsky O, Varcoe C (2007) From global to local and over the rainbow: Violence against women. In M. Morrow, O. Hankivsky, \& C. Varcoe (Editors), Women's health in Canada: Critical perspectives on theory and policy. University of Toronto Press, Toronto.

33. Guruge S, Khanlou N (2004) Intersectionalities of influence: researching the health of immigrant and refugee women. Can J Nurs Res 36: 32-47.

34. Thorne S, Reimer-Kirkham S, O'Flynn-Magee K (2004) The analytic challenge in interpretive description. International Journal of Qualitative Methods.

35. Bryman A (2001) Social research methods. Oxford University Press, New York.

36. Lincoln YS, Guba E (1985) Naturalistic inquiry. Newbury Park, CA: Sage.

37. Johnson MP (2006) Conflict and control: gender symmetry and asymmetry in domestic violence. Violence Against Women 12: 1003-18.

38. Hewamanne $S$ (2010) Stitching identities in a free trade zone: Gender and politics in Sri Lanka. University of Pennsylvania Press, Philadelphia.

39. Agocs C, Jain H (2001) Systemic racism in employment in Canada: diagnosing systemic racism in organizational culture. The Canadian Race Relations Foundation.

40. Chui T (2011) Women in Canada: A Gender-based Statistical Report. Statistics Canada.

41. Jayakoddy IL (2002) Gender identity and domestic violence in Sri Lanka: Reading experiences of violence through gender disclosure. Gothenburg University, Gothenburg.

42. Jayasuriya $\mathrm{V}$, Wijewardena $\mathrm{K}$, Axemo $\mathrm{P}$ (2011) Intimate partner violence against women in the capital province of Sri Lanka: prevalence, risk factors, and help seeking. Violence Against Women 17: 1086-1102.

43. Perera T, Gunawardane N, Jayasuriya V (2011) Review of Research Evidence on Gender Based Violence in Sri Lanka. Sri Lanka Medical Association, Colombo.

44. Regan L, Kelly L, Morris A, Dibb R (2007) "If only we'd known": An exploratory study of seven intimate partner homicides in Engleshire. Child and Woman Abuse Studies Unit, London Metropolitan University, London.

45. Schuler SR, Bates LM, Islam F (2008) Women's rights, domestic violence, and recourse seeking in rural Bangladesh. Violence Against Women 14: 326-345.

46. Mason R, Hyman I (2008) Intimate partner violence among immigrant and refugee women. In S Guruge \& E Collins (Editors), Working with Immigrant Women. Issues and strategies for mental health professionals. Centre for Addiction and Mental Health, Toronto.

47. Guruge S (2010) Perceptions of and responses to intimate partner violence: Findings from a study in the Sri Lankan Tamil community in Toronto. In J.S.F. Fong (Editors). Out of the Shadows: Woman Abuse in Ethnic, Immigrant, and Aboriginal Communities. Canadian Scholars Press: $264-283$

48. Hyman I, Mason R, Berman H, et al (2006) Perceptions of and responses to IPV among Tamil women in Toronto. Canadian Woman Studies 25:145-150.

49. Jayatunge, AAAVD (1998) 'Women and violence - a case study of Wendesiwatta settlement', Thesis submitted to the faculty of graduate studies, University of Colombo, in partial fulfillment of requirements for the degree of Master of Arts in Women's Studies.

50. Deraniyagala S (1992) An investigation into the incidence and causes of domestic violence in Colombo, Sri Lanka.

51. Dias M, Fernando D (1996) 'Women victims of violence; A study on admissions to a provincial general hospital', Fifth National Convention of Women's Studies, CENWOR, Colombo.

52. De Mel N, Peiris P, Gomez S (2013) Broadening gender: Why masculinities matter. Attitudes, practices and gender-based violence in four districts in Sri Lanka. Colombo : Care International Sri Lanka. 
Citation: Guruge S (2014) Perceptions About and Responses to Intimate Partner Violence in the Sinhalese Immigrant Community in Toronto. Arts Social Sci J S: S1-006. doi:10.4172/2151-6200.S1-006

Page 9 of 9

53. Jayaweera S, Sanmuga T, Dias M, Nagahawatte K (2010) A Study of Domestic Violence in Selected Locations in Sri Lanka. Colombo: CENWOR.

54. Guruge S (2007) The influence of gender, racial, social, and economic inequalities on the production of and responses to IPV in the postmigration context. Unpublished dissertation. University of Toronto, Toronto.

55. Morash M, Bui HN, Santiago AM (2000) Cultural-specific gender ideology and wife abuse in Mexican-descent families. International Review of Victimology 7: 67-91.

56. Tran CG, Des Jardins K (2000) Domestic violence in Vietnamese refugee and Korean immigrant communities. In J.L. Chin (Editors),
Relationships among Asian American women. American Psychological Association, Washington.

57. Yick AG (2001) Feminist theory and status inconsistency theory: Application to domestic violence in Chinese immigrant families. Violence Against Women 7: 545-562.

58. Naved RT, Azim S, Bhuiya A, Persson LA (2006) Physical violence by husbands: magnitude, disclosure and help-seeking behavior of women in Bangladesh. Soc Sci Med 62: 2917-2929.
This article was originally published in a special issue, entitled: "Violence against Immigrant Women: A Global Perspective throughout the LifeCycle", Edited by Nawal H Ammar, Arshia U Zaidi, University of Ontario Institute of Technology, Canada 